Synthèse bibliographique

\title{
HABITAT DE LA TRUITE COMMUNE (SALMO TRUTTA L.) PENDANT LA PÉRIODE JUVÉNILE EN RUISSEAU : PRÉFÉRENCES, MOUVEMENTS, VARIATIONS JOURNALIĖRES ET SAISONNIĖRES.
}

\author{
J.M. ROUSSEL (1), A. BARDONNET (2)
}

(1) INRA, UMR EQHC, Laboratoire d'Ecologie Aquatique, 65 rue de Saint-Brieuc, CS 84125, 35042 RENNES cedex, France. E-mail : roussel@ roazhon.inra.fr

(2) INRA, Unité de Recherche en Hydrobiologie, Equipe d'Ecologie Comportementale des Poissons, B.P. 3, 64310 SAINT-PEE-SUR-NIVELLE, France.

\section{RÉSUMÉ}

Cet article synthétise les résultats de travaux récents sur l'habitat de la truite commune pendant sa période juvénile en ruisseau. Par des approches in situ (affluents du Scorff, Bretagne) et en milieu expérimental, les variations temporelles d'utilisation de l'habitat ont été étudiées, ainsi que l'influence de certains facteurs abiotiques (vitesse de courant, profondeur, granulométrie, abris) et biotiques (prédation, compétition intraspécifique) sur les choix d'habitat de l'individu. A l'aide de la bibliographie sur le sujet, nous proposons un bilan des changements journaliers et saisonniers d'habitat du juvénile en ruisseau, depuis l'émergence jusqu'à la première reproduction. En matière de protection des milieux, l'accent est mis sur l'importance de la diversité des habitats disponibles dans les affluents où se reproduit l'espèce.

Mots-clés : truite, Salmo trutta, juvénile, ontogenèse, comportement, rythmes journaliers, habitat, mouvement.

THE HABITAT OF JUVENILE BROWN TROUT (SALMO TRUTTA L.) IN SMALL STREAMS: PREFERENCES, MOVEMENTS, DIEL AND SEASONAL VARIATIONS.

\begin{abstract}
This paper summarizes our recent investigations on habitat requirements for juvenile brown trout (Salmo trutta) in small tributaries of a lowland river system, the Scorff River (Brittany, France). Preferences for water velocity, depth, substrate and shelter, temporal variations in habitat use, and effects of predation and intra-specific competition on habitat choice, were studied both at field and laboratory. Using results from the literature, we propose a synthesis of the diel and seasonal shifts in habitat use by juvenile brown trout,
\end{abstract}


from the emergence up to the first spawning event. The role of instream habitat heterogeneity is emphasized for the management of such trout spawning tributaries.

Key-words : brown trout, Salmo trutta, parr, ontogeny, behaviour, diel rhythm, habitat preference, movement.

\section{INTRODUCTION}

La truite commune Salmo trutta L. est une espèce largement répandue sur l'ensemble du territoire français (ALLARDI et KEITH, 1991). Elle colonise les rivières où la température de l'eau est inférieure à $20^{\circ} \mathrm{C}$ en moyenne (exceptionnellement jusqu'à $25^{\circ} \mathrm{C}$ en été) et la saturation en oxygène dissous dépasse $80 \%$ (ELLIOTT, 1994). Espèce récréative très recherchée, elle a bénéficié par le passé de nombreux efforts visant à soutenir les effectifs par repeuplement (CHANGEUX, 1997). En parallèle, les recherches sur l'écologie de l'espèce se sont multipliées, suite aux travaux de LÉGER (1910) puis HUET (1954), sur le plan national et international.

Dans les années 1960-70, les rythmes d'activité et le régime alimentaire de l'animal furent particulièrement étudiés (notamment SWIFT, 1964 ; CHASTON, 1969 ; ELLIOTT, 1970 ; NEVEU, 1979), alors que l'occupation de l'espace et l'abondance des populations furent des thèmes porteurs dans les années 1970-80 (en France, on citera les travaux de HÉLAND, 1971 ; EUZENAT et FOURNEL, 1976 ; BAGLINIĖRE, 1979 ; NIHOUARN, 1983). A la fin des années 80 , sous l'impulsion nord-américaine, les recherches se sont orientées vers la modélisation des habitats hydrauliques (FRAGNOUD, 1987 ; SOUCHON et al., 1989 ; BELAUD et al., 1989). De nombreux développements ont suivi (notamment GINOT, 1995 ; POUILLY et al., 1995 ; BARAN, 1995 ; CAPRA, 1995 ; DELACOSTE, 1995 ; VALENTIN et al., 1996 ; LAMOUROUX, 1997 ; GOURAUD, 1999), plaçant les relations entre le poisson et son milieu au cœur des recherches actuelles sur les populations de truite.

En matière de gestion des populations, le recours systématique aux pratiques de repeuplement est actuellement remis en question (voir HOLL, 1997 ; CHANGEUX, 1997). On assiste à une ré-orientation des efforts de gestion en faveur de la protection des milieux naturels. Un tel objectif requiert une connaissance approfondie de la relation entre l'animal et le milieu où il évolue, pour identifier les habitats sensibles et méritant une attention particulière. Cependant, chez cette espèce, les variations temporelles de préférences d'habitat restent pratiquement inconnues, notamment pendant la période juvénile. Si des rythmes d'activité journaliers existent, comment se traduisent-ils en terme de déplacement dans l'habitat? Quels sont les mécanismes qui entrent en jeu ? Comment varient les préférences d'habitat durant l'ontogenèse, période où les besoins de l'individu changent rapidement?

En 1995, le Conseil Supérieur de la Pêche et l'Institut National de la Recherche Agronomique initiaient un programme de recherche sur le sujet (conventions INRA/CSP n`95372 et 99265). Pendant quatre années (1995-1998), des investigations ont été menées en milieu naturel, semi-naturel et contrôlé, pour mieux comprendre les relations fonctionnelles entre le juvénile et son habitat en ruisseau. Les recherches ont été organisées autours de quatre thèmes principaux : 1/ rythmes journaliers d'utilisation de l'habitat par l'alevin émergent, 2/ variations spatiales et temporelles de densités des juvéniles en ruisseau, $3 /$ habitats d'alimentation et de repos du juvénile d'un an et plus, et 4/ compétition intra-spécifique et rôle des abris chez les juvéniles d'un an et plus. En associant les principaux résultats à la littérature existante, le présent article propose un schéma récapitulatif de l'utilisation de l'habitat par la truite commune lors de sa période juvénile en ruisseau. 


\section{ÉMERGENCE ET RYTHMES D'UTILISATION DE L'HABITAT PAR LES ALEVINS}

La reproduction de la truite se déroule en rivière à la fin de l'automne ou au début de l'hiver, les œufs étant enfouis sous les graviers par la femelle. Dans les ruisseaux du Massif Armoricain, les frayères se situent dans des habitats peu profonds (moins de $30 \mathrm{~cm}$ de profondeur), courants et à granulométrie grossière (éléments de 2 à $5 \mathrm{~cm}$ de diamètre, travaux de EUZENAT et FOURNEL, 1976; NIHOUARN, 1983). Le développement embryonnaire a lieu dans le substrat, et concerne les stades embryon (avant éclosion) et embryon libre (après éclosion, sensu BALON, 1975). Lorsque ses réserves vitellines arrivent à épuisement, l'alevin émerge des graviers et entame sa période juvénile, qui se terminera avec la première maturation sexuelle. II est alors confronté à deux impératifs vitaux qui vont déterminer son placement dans l'habitat (HÉLAND et al., 1995) : se maintenir dans l'environnement lotique, et s'alimenter en évitant les prédateurs.

\section{Expérimentation}

Au laboratoire (installations INRA, Rennes), des canaux lotiques $(4 \times 0,5 \times 0,3 \mathrm{~m})$ ont été aménagés de sorte à créer un habitat de berge (profondeur $2 \mathrm{~cm}$, vitesse de courant 0 à $2 \mathrm{~cm} / \mathrm{s}$ ), et un habitat de chenal (profondeur $12 \mathrm{~cm}$, vitesse de courant 2 à $4 \mathrm{~cm} / \mathrm{s}$ ), berge et chenal étant séparés par une pente. Des lots d'alevins vésiculés d'origine sauvage ont été successivement installés sous les graviers, côté chenal, l'expérience étant réalisée deux fois à l'identique en présence ou non de prédateurs (Cottus gobio L.) ou de proies dans la dérive. Dans chaque cas, les observations comportementales ont été réalisées pendant 6 jours à partir de l'émergence (pour plus de détails, voir ROUSSEL et BARDONNET, 1999). Les résultats montrent qu'un à deux jours après le début de la phase d'émergence, un rythme nycthéméral d'utilisation de l'habitat se met en place, qu'il y ait ou non des proies dans la dérive. D'une manière générale, les alevins maintiennent un poste en nage statique face au courant dans le chenal de jour, puis se posent sur le gravier en berge la nuit (Figure 1). En présence de chabots, la plupart des alevins reste cachée sous les graviers, les individus visibles se tenant préférentiellement en berge de jour comme de nuit.

\section{Discussion}

Chez la truite commune, l'émergence des graviers et la dévalaison précoce associée sont des phénomènes typiquement nocturnes (BARDONNET et al., 1993). Ainsi, l'enjeu immédiat pour l'alevin émergent serait de rejoindre les habitats de berge, qui offrent à la fois un refuge vis-à-vis du courant et contre les prédateurs d'origine aquatique. En milieu naturel, les alevins de plusieurs espèces de salmonidés fréquentent ces habitats de bordure : Salmo clarki (MOORE et GREGORY, 1988), Salvelinus fontinalis (WALSH et al., 1988), Thymallus thymallus (BARDONNET et al., 1991), Oncorhynchus mykiss (KOCIK et TAYLOR, 1996). Chez Salmo trutta, HUBERT et al. (1994) remarquent que le nombre d'alevins de taille comprise entre 23 à $48 \mathrm{~mm}$ (Longueur Fourche) baisse sensiblement pendant la journée dans les habitats de bordure, indiquant que les déplacements entre berge et chenal se prolongent plusieurs semaines après l'émergence.

L'alimentation sur dérive étant le mode de nutrition privilégié pendant toute la période juvénile (CHASTON, 1969 ; ELLIOTT, 1970 ; HUBERT et RHODES, 1992), les juvéniles vont peu à peu se poster dans des habitats plus courants et profonds de jour (KOCIK et TAYLOR, 1996), à mesure qu'ils grandissent et que leurs performances de nage s'améliorent. Une fois la taille de $50 \mathrm{~mm}$ (LF) atteinte, ils semblent abandonner progressivement les habitats de bordure la nuit (LAVOIE et HUBERT, 1996), et la hauteur d'eau devient un paramètre essentiel du placement des juvéniles. Cet aspect a été étudié dans la suite du travail par le biais d'inventaires en milieu naturel. 

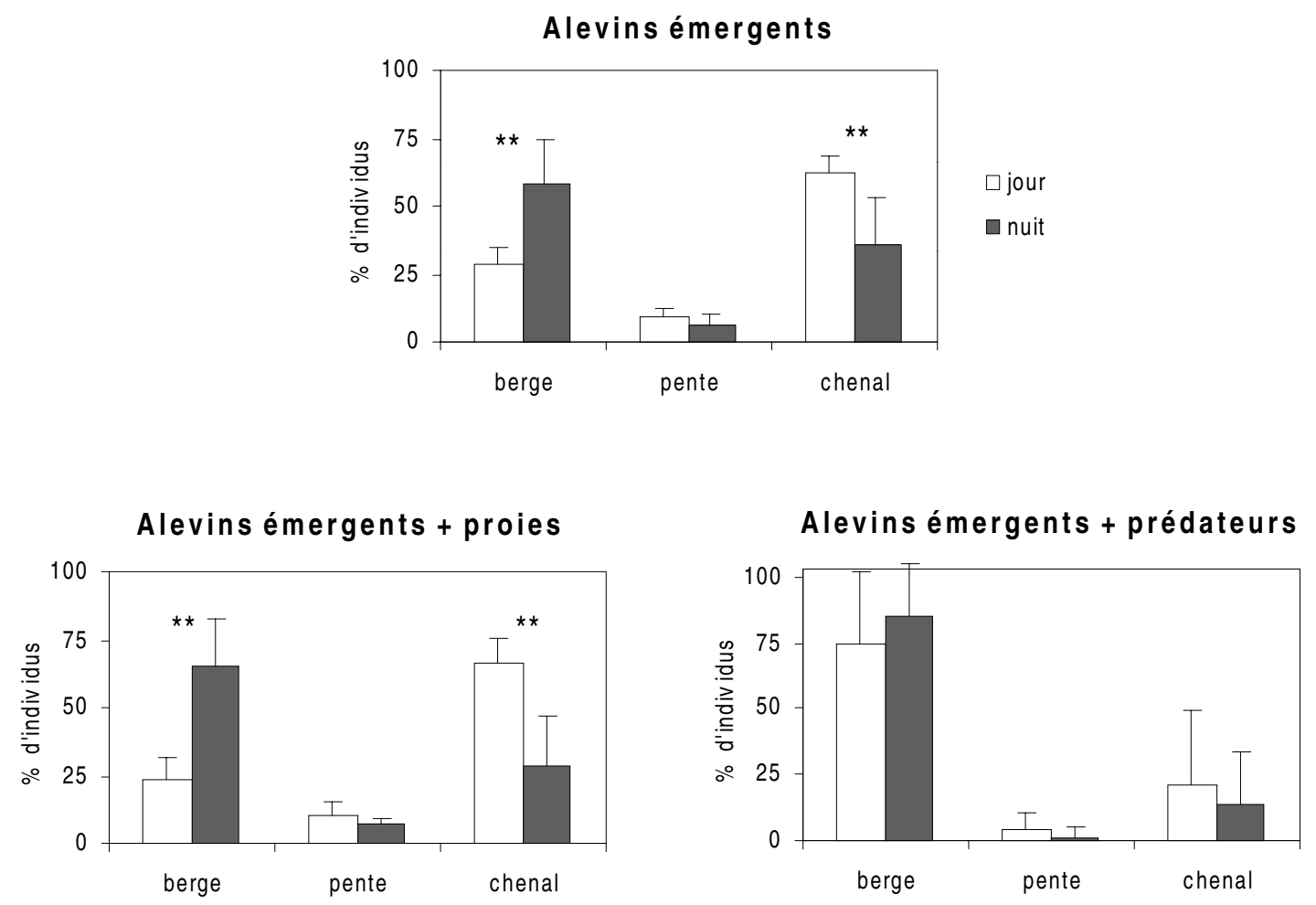

Figure 1

Pourcentage moyen d'occupation de la berge, de la pente et du chenal par les alevins, sur une période de 6 jours suivant le début de l'émergence. Chaque situation expérimentale présentée (alevins seuls, alevins + proies, alevins + prédateurs) correspond aux observations réalisées sur 2 canaux expérimentaux, contenant 60 individus chacun. Les traits verticaux indiquent les écarts types ; les différences jour/nuit sont évaluées par des tests de Mann-Whitney $\left(^{*}: p<0,05 ;{ }^{* *}: p<0,01\right)$. Modifié d'après ROUSSEL et BARDONNET (1999).

Figure 1

Spatial and temporal distribution of emerging brown trout in experimental channels. Observations were made on two channels (60 alevins each), in a period of 6 days following the emergence start. Three treatments were performed: alevins only (alevins émergents), alevins and drifting preys (alevins émergents + proies), alevins and predators Cottus gobio (alevins émergents + prédateurs). Y-axis: mean percentages of alevins in the margin (berge), the slope (pente) and the deep habitat (chenal), during daylight (open bars) and at night (filled bars). Vertical lines represent standard deviations, and significant diel differences (MannWhitney tests) are given as ${ }^{*}$ when $p<0,05$ and ${ }^{* *}$ when $p<0,01$. Modified from ROUSSEL and BARDONNET (1999). 


\section{VARIATIONS TEMPORELLES DES DENSITÉS DE JUVÉNILES EN RUISSEAU}

Dans de nombreuses rivières européennes, les densités de juvéniles de l'année sont fortes dans les habitats peu profonds (inférieurs à $20 \mathrm{~cm}$ ) et courants de type radier, les juvéniles plus âgés fréquentant majoritairement les profondeurs supérieures à 20 ou $30 \mathrm{~cm}$ (JONES, 1975 ; SOLOMON et TEMPLETON, 1976 ; BOHLIN, 1977 ; KARLSTRÖM, 1977 ; BAGLINIÈRE et CHAMPIGNEULLE, 1982 ; EGGLISHAW et SHACKLEY, 1982 ; NIHOUARN, 1983 ; KENNEDY et STRANGE, 1986 ; HEGGENES, 1988). Dans les cours d'eau peu pentus du Massif Armoricain (pente généralement inférieure à $1 \%$ ), la séquence radier/profond est une unité typique de la géomorphologie du chenal (voir BISSON et MONTGOMERY, 1996). Elle offre, de ce point de vue, un milieu fort contrasté et susceptible de supporter les variations temporelles d'utilisation de l'habitat par les juvéniles de truite.

\section{Expérimentation}

Sur trois séquences radier/profond du ruisseau de Kerlégan, affluent de la partie basse du Scorff (Morbihan), les variations journalières et saisonnières de densités des juvéniles ont été suivies par pêche électrique pendant une année. L'utilisation de filets spéciaux a permis d'éliminer le risque de fuite des poissons (pour plus de détails, voir ROUSSEL et BARDONNET, 1997). Après la chute des filets dans l'eau, deux à trois passages sont réalisés en pêche électrique, en vue d'estimer les densités de juvéniles par la méthode des maximums de vraisemblance (ZIPPIN, 1956). Quatre campagnes de pêche ont été réalisées en juin 1995, octobre 1995, janvier 1996 et avril 1996, chacune comprenant des inventaires de jour et de nuit.

\section{Discussion}

Durant la période étudiée, le débit du ruisseau a varié d'environ $30 \mathrm{l} / \mathrm{s}$ en octobre à plus de $300 \mathrm{l} / \mathrm{s}$ en janvier. Après l'émergence (avril 96), les densités de $0+$ sont deux à trois fois plus fortes en radier qu'en profond (Figure 2). Malgré les faibles effectifs pêchés en 1995 (faible recrutement cette année là), la tendance s'inverse au cours de l'été, les densités de $0+$ devenant plus fortes en profond qu'en radier en octobre. Pendant les forts débits hivernaux, les profondeurs en radier étant supérieures à $20 \mathrm{~cm}$, les densités de $0+$ semblent s'équilibrer entre radier et profond. On n'enregistre aucune variation nycthémérale significative entre densités de $0+$ en radier et en profond.

Pour les truites d'un an et plus (Figure 2), les densités chutent en janvier sur le secteur d'étude. Ceci peut s'expliquer par un phénomène de migration à une plus large échelle des individus précoces au moment de la reproduction (maturation sexuelle possible dès 1+ dans le Massif Armoricain, voir BAGLINIËRE et al. 1987). En avril, on assiste à une sensible augmentation numérique de individus d'un an et plus sur le secteur, ce réajustement des densités se faisant probablement à la faveur des mouvements de juvéniles d'un été depuis la zone amont et les sous-affluents (BAGLINIĖRE et al., 1989).

Pendant la période estivale (juin et octobre), 40 à $60 \%$ des juvéniles d'un et deux ans est capturé en radier la journée (profondeurs moyennes inférieures à $10 \mathrm{~cm}$, vitesses moyennes comprises entre 20 et $50 \mathrm{~cm} / \mathrm{s}$ ). De nuit au contraire, la quasi totalité des juvéniles est capturée en profond, où les densités peuvent dépasser $30 \mathrm{ind} / \mathrm{m}^{2}$. Des marquages recaptures par PIT-tag ont également montré que le taux de sédentarité dans les séquences radier/profond est plus fort en été lorsque les débits sont faibles. Les variations journalières des densités en radier et profond suggèrent qu'un rythme nycthéméral d'utilisation de la séquence radier/profond se met alors en place, en relation avec les activités d'alimentation, de repos ou de cache du juvénile d'un an et plus. Cette hypothèse a fait l'objet de tests par la suite, grâce à des observations en plongée dans le milieu naturel. 
Densités de 0+ en profond

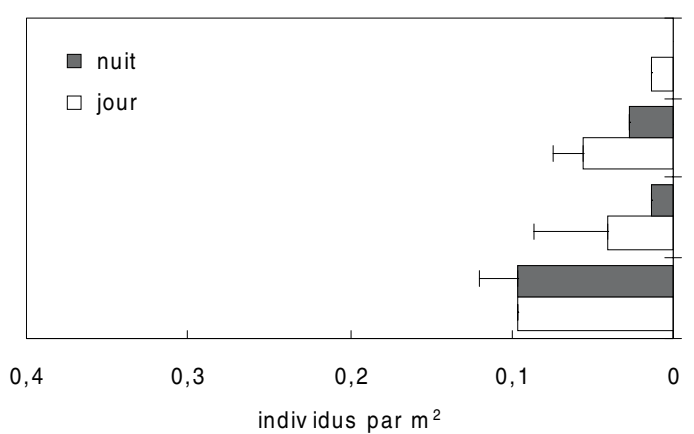

Densités de 1+et 2+en profond

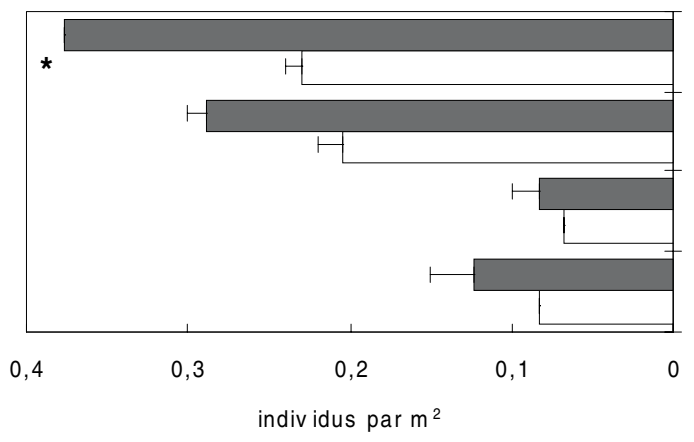

Densités de 0 + en radier

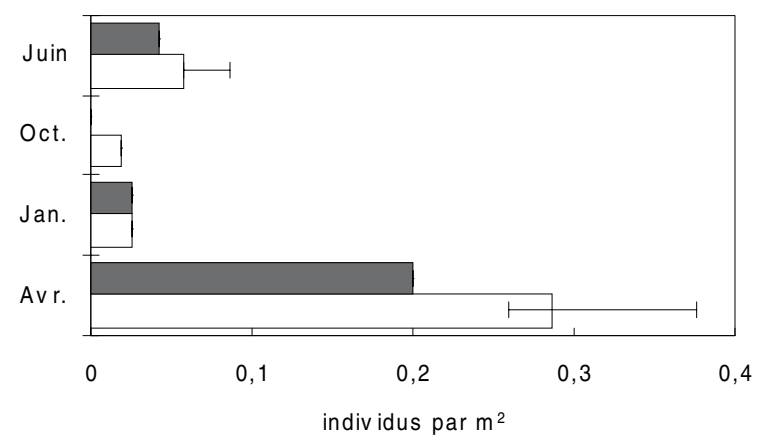

Densités de 1+ et 2+en radier

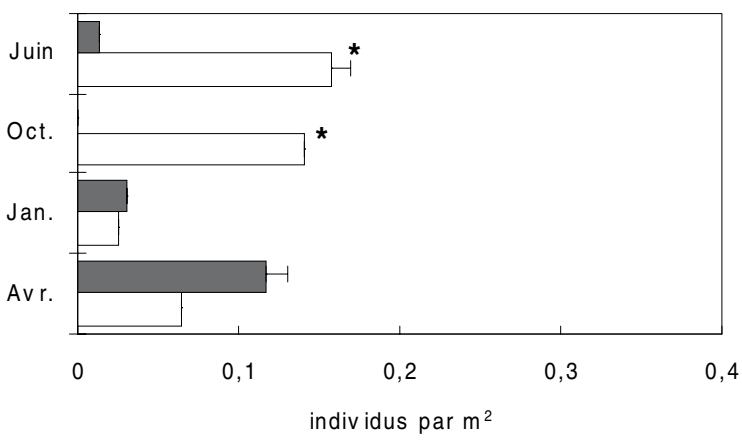

Figure 2

Variations journalières et saisonnières des densités de juvéniles de l'année (0+) et plus âgés (1+ et 2+), calculées à partir des inventaires en pêche électrique réalisés sur 3 séquences radier/profond du ruisseau de Kerlégan (Scorff, Morbihan). Les densités ont été estimées par la méthode de Zippin (2 ou 3 passages), excepté dans les cas où tous les poissons ont été capturés en premier passage, les traits horizontaux représentant les intervalles de confiance à $95 \%$. Les différences jour/nuit sont évaluées par des tests de Mann-Whitney $\left(^{*}: p<0,05\right)$. Modifié d'après ROUSSEL et BARDONNET (1997).

Figure 2

Diel and seasonal variations in densities of young-of-the-year $(0+)$ and older juveniles (1+ and 2+), in riffles (radier) and pools (profond) of Kerlégan Brook (Scorff River, Brittany, France). Three riffle-pool sequences were electrofished during daylight (open bars) and at night (filled bars), in June 1995 (Juin), October 1995 (Oct.), January 1996 (Jan.), April 1996 (Avr.). The densities were estimated by the Zippin' method ( 2 or 3 sweeps) except when all fish were caught in sweep \#1, horizontal lines representing the $95 \%$ confident level. Significant diel differences are indicated (Mann-Whitney test, ${ }^{*}: p<0,05$ ). Modified from ROUSSEL and BARDONNET (1997). 


\section{HABITATS D'ALIMENTATION ET DE REPOS DES JUVÉNILES D'UN AN ET PLUS}

Le déroulement des activités quotidiennes chez les poissons se traduit par l'alternance de période de recherche de nourriture et de période d'inactivité, au cours de laquelle s'expriment les comportements de repos, de confort ou de cache (HELFMAN, 1993). La succession jour/nuit est alors un facteur environnemental qui rythme l'activité (MÜLLER, 1978). Chez la truite commune, le rythme paraît très flexible, si l'on en juge par des résultats souvent divergents dans la littérature. Le rythme journalier peut se modifier au cours des saisons, comme l'a montré expérimentalement ERIKSSON $(1973,1978)$. Sous nos latitudes, OVIDIO (1999) rapporte une activité plutôt diurne en hiver et nocturne en été chez l'adulte. Pour des latitudes plus septentrionales, la truite diurne ou crépusculaire en été deviendrait plus exclusivement nocturne en hiver (HEGGENES et al., 1993).

Le rythme journalier peut aussi se modifier au cours de l'ontogenèse. Hormis le comportement de dévalaison post-émergence, l'activité de l'alevin semble typiquement diurne (PEREZ, 1986 ; ROUSSEL et BARDONNET, 1999), alors que l'adulte est plus nettement nocturne (CLAPP et al., 1990 ; YOUNG et al., 1997 ; YOUNG, 1999) ou crépusculaire (BACHMAN et al., 1979 ; OVIDIO, 1999). Pendant la période juvénile, les truitelles peuvent être diurnes (ROUSSEL et BARDONNET, 1995) ou nocturnes (CHASTON, 1968), probablement en relation avec la disponibilité des proies dans le milieu (CHASTON, 1969 ; ELLIOTT, 1970). Quelles que soient les modalités de son expression, le rythme nycthéméral doit se traduire par des mouvements en direction d'habitats où les conditions mésologiques sont les plus propices au comportement à réaliser, alimentation ou repos/cache.

\section{Expérimentation}

Des observations en plongée ont été réalisées pour identifier les habitats quotidiens des juvéniles d'un an et plus, sur un affluent de la partie basse du Scorff, le ruisseau de Saint-Sauveur. Un secteur de $110 \mathrm{~m}$ de long (largeur $3 \mathrm{~m}$ environ), composé de successions de radier, plats et profonds, a été prospecté durant l'étiage en août 1997 (débit $110 \mathrm{l} / \mathrm{s}$ ), de jour et de nuit. L'observation individuelle de chaque poisson a permis d'en déterminer l'activité (alimentation ou repos), sa position exacte étant reportée sur une carte (pour plus de détails, voir ROUSSEL et al., 1999). L'habitat utilisé (profondeur, granulométrie, vitesse de courant et type de faciès) a ensuite été mesuré puis comparé à l'habitat disponible sur le secteur.

Pendant la période d'étude, tous les juvéniles d'un an et plus sont en alimentation sur la dérive de jour (43 individus observés), et $95 \%$ sont au repos de nuit (52 individus observés). De nettes variations de préférences d'habitat sont enregistrées entre le jour et la nuit : de jour, les juvéniles se postent dans des microhabitats courants (15 à $45 \mathrm{~cm} / \mathrm{s}$ ) au substrat grossier ( 2 à $25 \mathrm{~cm}$ de diamètre) et aux profondeurs moyennes (entre 25 et $45 \mathrm{~cm}$ ). De nuit par contre, ils préfèrent les microhabitats lents ( 0 à $15 \mathrm{~cm} / \mathrm{s}$ ), au substrat fin (limon et sable) et situés dans les plus fortes hauteurs d'eau du secteur (40 à $55 \mathrm{~cm}$ ). La ségrégation spatiale des microhabitats diurnes et nocturnes se retrouve à l'échelle géomorphologique du faciès d'écoulement (Figure 3). Les postes favorables à l'alimentation sont localisés dans les faciès courants (radier, plat courant) et, dans une moindre mesure, dans les veines de courant en profond, alors que les postes de repos nocturnes sont regroupés en profond. 


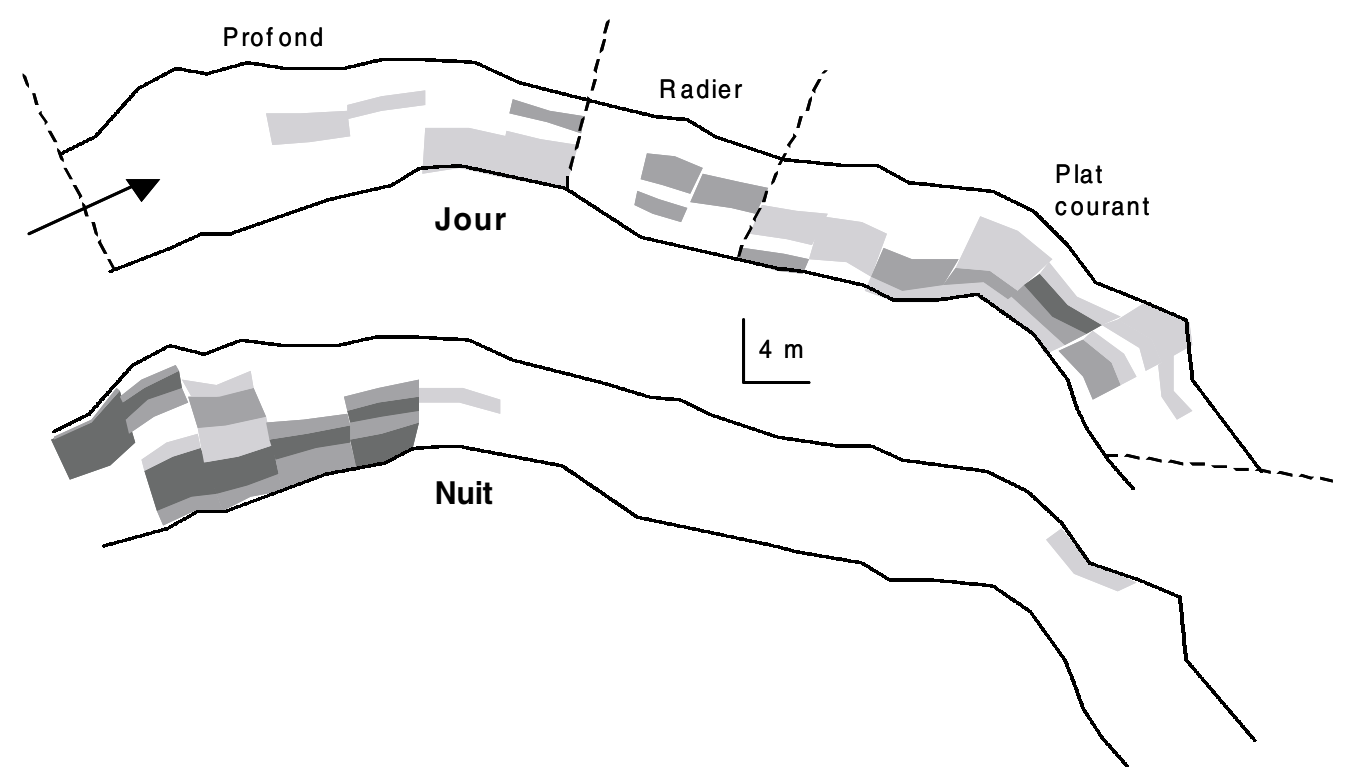

Figure 3

Distribution spatiale des microhabitats favorables aux juvéniles d'1 et 2 ans, de jour et de nuit, sur une séquence profond/radier/plat-courant du ruisseau de Saint-Sauveur (Scorff, Morbihan). La flèche indique le sens général d'écoulement du ruisseau, le débit étant de $110 \mathrm{l} / \mathrm{s}$ (étiage). Les calculs ont été réalisés à partir des préférences d'habitat établies sur le ruisseau, et à l'aide du logiciel EVHA : plus l'habitat est favorable, plus il apparaît foncé sur le schéma. Modifié d'après ROUSSEL et al. (1999).

\section{Figure 3}

Spatial distribution of suitable habitats for juveniles (age-1 and age-2) that feed on drift during daylight (Jour) and rest at night (Nuit), in a pool/riffle/run sequence (profond/radier/plat courant) of Saint-Sauveur Brook (Scorff River, Brittany, France). The stream discharge was $110 \mathrm{l} / \mathrm{s}$ during the experiment in Summer 1997. The arrow indicates the main flow direction. Using local habitat suitability criteria for depth, velocity and substrate, the habitat on the site was evaluated using the EVHA software : the suitability increases from white (unsuitable) to dark-grey (maximum suitability) shading. Modified from ROUSSEL et al. (1999).

\section{Discussion}

II existe dans la littérature plusieurs études sur la modélisation bio-énergétique de l'habitat d'alimentation sur dérive chez les salmonidés. Dans ce mode d'alimentation où l'énergie parvient à l'animal par entraînement passif, le nombre de proies disponibles par unité de temps est théoriquement proportionnel à la vitesse du courant (FAUSCH, 1984 ; HILL et GROSSMAN, 1993). En conséquence, un microhabitat trop lent serait limitant en terme d'apport énergétique (nombre de proies insuffisant), alors qu'un habitat trop rapide nécessiterait une grande dépense d'énergie pour maintenir le poste (FACEY et GROSSMAN, 1992) et/ou réduirait la capturabilité des proies (HUGHES et DILL, 1990 ; HILL et GROSSMAN, 1993). 
Dans le ruisseau de Saint-Sauveur, la vitesse focale préférée par les juvéniles d'un an et plus est de $22 \mathrm{~cm} / \mathrm{s}$ (Figure 4), ce qui est très proche de la valeur énergétique idéale proposée par HILL et GROSSMAN (1993) chez le juvénile d'Oncorhynchus mykiss. Pour Salmo trutta, les observations en milieu naturel qui relient de façon certaine alimentation sur dérive et préférence d'habitat semblent corroborer ce résultat : les vitesses varient de 10 à $40 \mathrm{~cm} / \mathrm{s}$ pour les 1+ (GOSSE et HELM, 1982 ; SHULER et NEHRING, 1994) et de 20 à $50 \mathrm{~cm} / \mathrm{s}$ pour les truites plus âgées (GOSSE et HELM, 1982 ; SHIRVELL et DUNGEY, 1983 ; HAYES et JOWETT, 1994 ; SHULER et NEHRING, 1994).

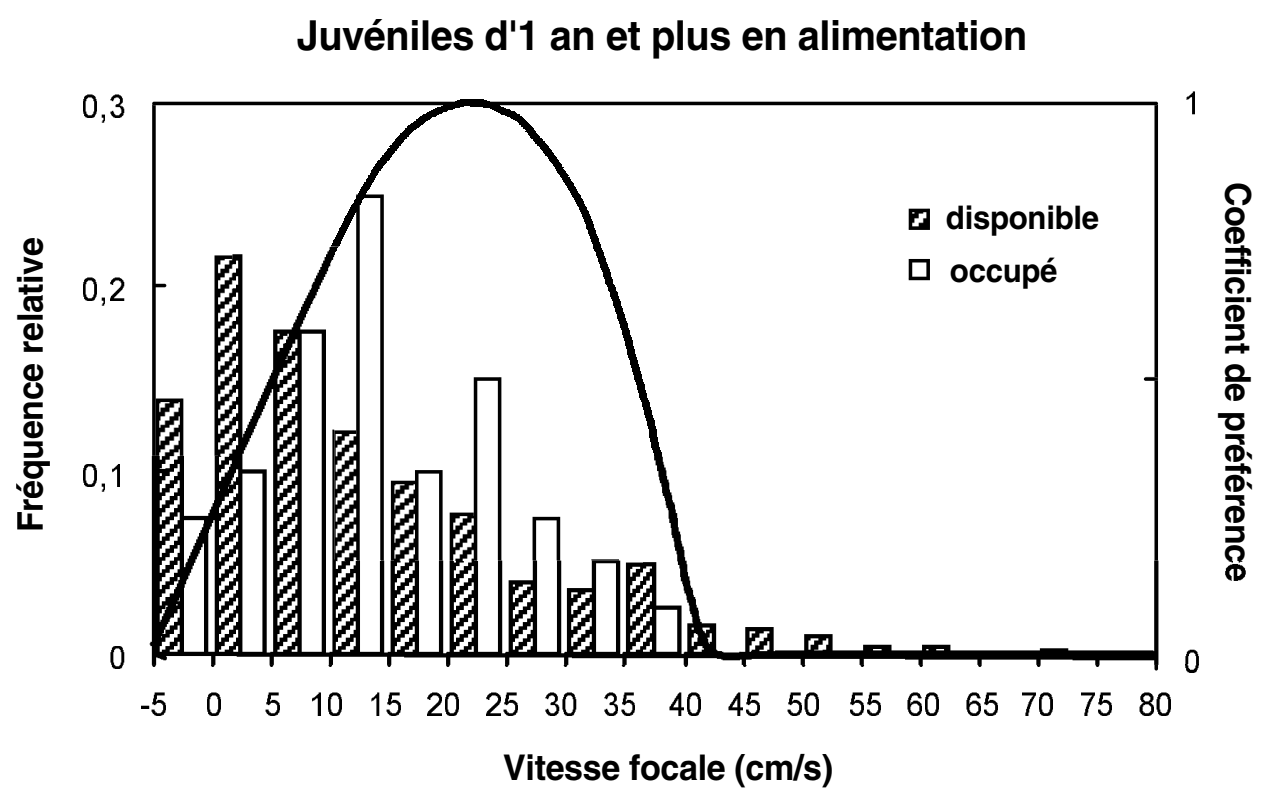

\section{Figure 4}

Vitesses focales sélectionnées par les juvéniles d'1 et 2 ans en alimentation sur dérive, dans le ruisseau de Saint-Sauveur (Scorff, Morbihan). La préférence (courbe noire) est calculée en comparant vitesses disponibles et vitesses occupées sur le secteur, mesurées au point focal du poisson (43 individus observés en plongée). Modifié d'après ROUSSEL et al. (1999).

Figure 4

Focal velocity preference for juveniles (age-1 and age-2) when feeding on drift in Saint-Sauveur Brook (Scorff River, Brittany, France) in Summer 1997 (stream discharge $110 \mathrm{l} / \mathrm{s}$ ). Forty three fish were observed on the site by snorkelling, the focal velocity use (open bars) was recorded and compared to mean velocity available (hatched bars) to calculate habitat suitability criteria (solid line). Modified from ROUSSEL et al. (1999).

Dans les ruisseaux étudiés, les vitesses de courant favorables à l'alimentation se rencontrent essentiellement en radier ou plat courant. Lors des faibles débits estivaux, ces faciès jouent un rôle d'habitat trophique pour le juvénile d'un an et plus. La séquence radier/profond semble être une aire d'activité journalière (sensu BARAS, 1992) privilégiée, qui regroupe les conditions de gîte (en profond) et de nutrition (en radier). Dans des ruisseaux comparables en Irlande, BRIDCUT et GILLER (1993a ; 1993b) analysant le régime alimentaire de la truite commune, concluent qu'une partie des juvéniles présents dans les profonds se déplacent en radier pour capturer les larves d'invertébrés qui 
dérivent. D'un point de vue quantitatif, BARAN et al. (1997) montrent que dans les cours d'eau de montagne, les densités de truites en profond sont d'autant plus fortes que ces faciès sont localisés à proximité d'habitats courants de type radier.

Ainsi, la sélection d'un habitat de gîte par les juvéniles et sa fréquentation pourraient être conditionnées par la proximité d'un habitat de nourrissage, comme c'est le cas chez le barbeau fluviatile Barbus barbus (BARAS, 1997). Les juvéniles de truite peuvent se regrouper en profond pendant les phases de repos nocturne, la territorialité et la compétition spatiale s'exprimant moins que pendant les périodes d'alimentation. Des études ont été entreprises par la suite pour évaluer les effets de la structuration de l'habitat en radier sur l'accès aux postes d'alimentation favorables.

\section{COMPÉTITION POUR LES POSTES D'ALIMENTATION ET RÔLE DES ABRIS}

L'existence d'un territoire et sa défense vis-à-vis des congénères impliquent que la densité des juvéniles peut être limitée par la taille des territoires (GRANT et KRAMER, 1990 ; KEELEY et GRANT, 1995 ; STEINGRIMSSON et GRANT, 1999). Pour la truite commune, les comportements agonistiques qui apparaissent quelques jours après l'émergence conduisent rapidement à la stabilisation territoriale des individus (HÉLAND, 1971, 1977).

En référence à la théorie d'une recherche optimale de nourriture (optimal foraging theory, MacARTHUR et PIANKA, 1966 ; GERKING, 1994), le poste le plus intéressant à défendre offre le meilleur compromis entre l'énergie gagnée par ingestion de proies et celle dépensée pour leur obtention. II existe alors une relation positive entre le rang social de l'individu et la profitabilité du poste qu'il occupe (FAUSCH, 1984 ; HUGHES, 1992), le meilleur poste étant l'assurance d'une croissance plus rapide et d'un meilleur succès reproducteur. Dans le contexte de la séquence radier/profond présentée précédemment, les postes les plus favorables à l'alimentation sur dérive doivent donc être occupés prioritairement par les individus dominants.

\section{Expérimentation}

Le partage des postes au sein de la hiérarchie et l'incidence de la structuration de l'habitat ont été étudiés dans un chenal artificiel à ciel ouvert possédant deux chambres d'observation subaquatiques (installations INRA, Saint-Pée sur Nivelle). Les conditions trophiques dans le chenal étaient proches d'une situation naturelle (dérive d'origine endogène et exogène). Deux séquences radier/profond de 8,5 et $12 \mathrm{~m}^{2}$ closes par des grillages (maille $1 \mathrm{~cm}$ ) ont été recréées par aménagement des galets du fond, une devant chaque chambre d'observation. Dans chaque séquence, le radier est placé à l'amont (profondeur $14 \mathrm{~cm}$, vitesse moyenne $12 \mathrm{~cm} / \mathrm{s}$ ), une pente faisant la transition avec le profond situé à l'aval (profondeur $53 \mathrm{~cm}$, vitesse moyenne nulle au fond et $4 \mathrm{~cm} / \mathrm{s}$ en surface). Plusieurs groupes de juvéniles de truite capturées en milieu naturel (LF comprise entre 112 et $133 \mathrm{~mm}$ ) ont été successivement mis en situation en août et septembre 1996, en appliquant une densité de $0,85 \mathrm{ind} / \mathrm{m}^{2}$ dans chaque séquence (pour plus de détails, voir ROUSSEL, 1998).

En prenant des groupes de poissons naïfs à chaque fois, 3 types d'aménagement en radier ont été testés : 1) sans abri, 2) avec abris émergés (plaques opaques maintenues au dessus de l'eau) fournissant un simple ombrage, et 3 ) avec abris immergés (lanières de plastiques ondulant dans le courant) fournissant un isolement visuel entre les congénères. Les variations de fréquentation du radier en fonction du type d'abri ont été testées selon la méthode des probabilités exactes de Fisher (SIEGEL et CASTELLAN, 1988), les situations expérimentales (radier sans abri, avec abris émergés ou avec abris immergés) étant testées deux à deux. 

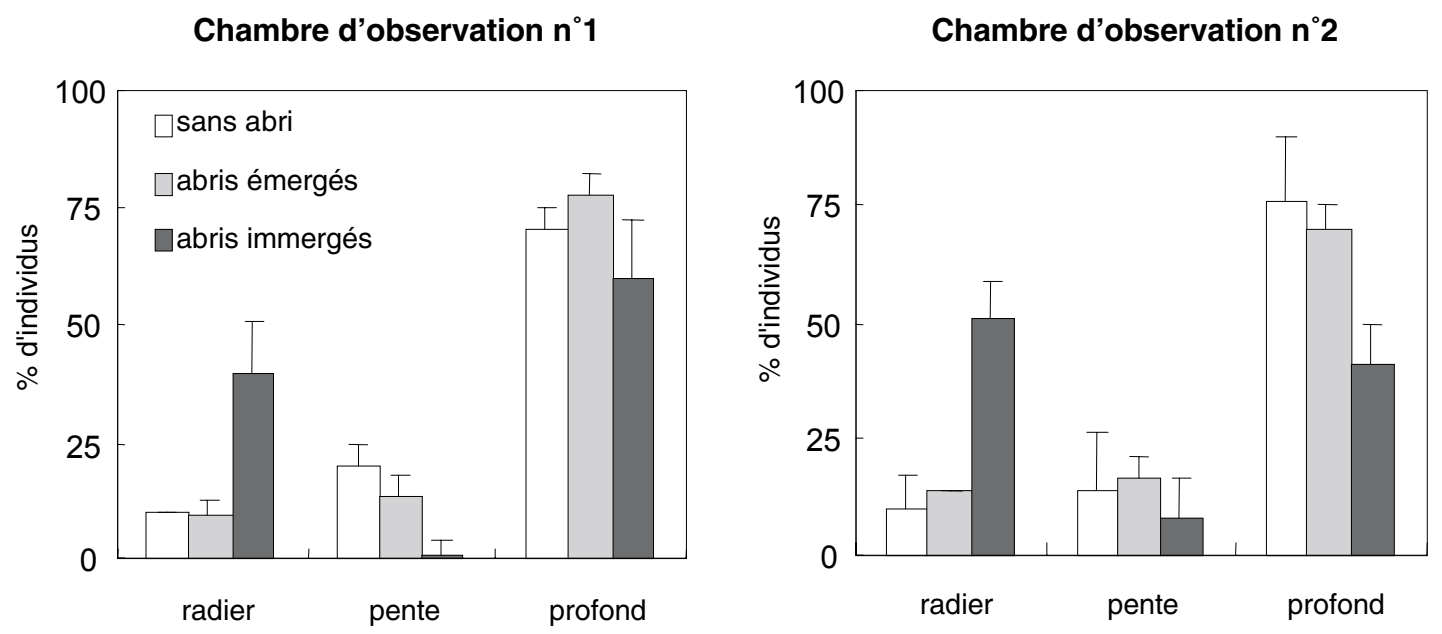

Figure 5

Répartition diurne des juvéniles (groupes de 7 ou 10 individus, $112 \mathrm{~mm}<\mathrm{LF}<133 \mathrm{~mm}$ ) dans 2 séquences radier/profond reconstituées en ruisseau expérimental, en fonction du type d'abri disposé en radier. Pour chaque situation expérimentale (pas d'abri, abris émergés, ou abris immergés), les fréquentations moyennes du radier, de la pente et du profond, sont calculées sur la base de trois relevés de position quotidiens réalisés pendant 3 jours consécutifs. Les traits verticaux correspondent aux écarts types.

\section{Figure 5}

Diurnal distribution of juveniles (groups of 7 or $10 \mathrm{fish}, 112 \mathrm{~mm}<\mathrm{FL}<133 \mathrm{~mm}$ ) in an experimental outdoor channel. The channel was equipped with 2 full-view windows on one side from which to observe the distribution and behaviour of fish. A riffle/pool unit was designed in front of each lateral window (riffle upstream). Three treatments were done: no shelter (open bars), overhead cover in riffle (light-grey bars), or submerged shelters in riffle (dark-grey bars) that provide concealment (plastic ribbons). Numbers of fish in riffle (radier), pool (profond) and the transition between these 2 habitats (pente), were recorded 3 times a day on 3 consecutive days. Y-axis: mean percentages of fish in each habitat (vertical lines $=$ standard deviations).

En l'absence d'abri, la fréquentation du radier est de $10 \%$ en moyenne dans les deux séquences radier/profond (Figure 5). L'organisation hiérarchique du groupe révèle la présence d'un dominant territorial posté à l'amont en radier, emplacement lui donnant l'exclusivité d'accès aux proies dérivant de l'amont. Dans le profond, les poissons sont contraints à des densités très fortes, certains individus alternent un comportement d'alimentation sur dérive et sur le fond, d'autres ne sont jamais territoriaux. En présence d'abris émergés (Figure 5), la répartition des individus est inchangée (test de Fisher, $p>0,05)$. 
A l'inverse, la présence d'abris immergés (Figure 5) entraîne une augmentation significative de la fréquentation du radier (test de Fisher, $p<0,05$ ) dans les deux séquences radier/profond. Environ la moitié des juvéniles (40 à $50 \%$ ) établit un poste d'alimentation sur dérive en radier, dont l'accès n'est plus verrouillé par le dominant. On note alors que tous les poissons sont territoriaux, et on enregistre dans le même temps une modification sensible des interactions agonistiques au sein des groupes de juvéniles.

\section{Discussion}

L'importance du facteur abri sur le placement individuel de la truite (voir par exemple, BUTLER et HAWTHORNE, 1968 ; DEVORE et WHITE, 1978 ; CUNJAK et POWER, 1987 ; MESICK, 1988 ; MÄKI-PETÄYS et al., 1997), et sur les densités de population (voir LEWIS, 1969 ; MORTENSEN, 1977 ; NIELSEN, 1986 ; WESCHE et al., 1987 ; LIM et al., 1993 ; BARAN et al., 1995 ; MARIDET et SOUCHON, 1995), est abondamment décrite dans la littérature. On sait que la truite affectionne particulièrement les structures immergés offrant un abri tactile (DEVORE et WHITE, 1978), c'est-à-dire contre lequel elle peut se dissimuler des prédateurs. $A$ ce titre, la végétation aquatique de certaines rivières peut jouer un rôle particulier (HAURY et BAGLINIĖRE, 1990, 1996). Nos résultats suggèrent que la présence d'abris immergés permet en outre une meilleure accessibilité aux postes d'alimentation sur dérive pour les individus dominés. Lorsqu'à l'étiage, les conditions hydrauliques sont peu favorables à l'alimentation sur dérive du fait des faibles vitesses de courant, les abris immergés joueraient un rôle dans le partage des postes, en facilitant le recouvrement des territoires défendus par chaque individu.

Des observations complémentaires réalisées par enregistrement vidéo infra-rouge ont également montré que certains individus sont actifs et s'alimentent de nuit en radier. L'aptitude à capturer des proies la nuit est connue chez la truite (ROBINSON et TASH, 1979). Un partage temporel des postes d'alimentation serait également possible entre les congénères, certains individus accédant à un poste d'alimentation favorable la nuit uniquement (ROUSSEL, 1998). De plus, GLOVA et FIELD-DODGSON (1995) observent que les individus peuvent alterner phases d'alimentation et de repos plusieurs fois pendant la journée. Ces rythmes, en relation avec les mécanismes de satiété et les besoins physiologiques de l'animal, pourraient ainsi faciliter l'alternance des individus sur les postes d'alimentation en radier.

\section{BILAN DE L'UTILISATION DE L'HABITAT PAR LE JUVÉNILE EN RUISSEAU}

L'ensemble des expériences réalisées permet de dresser le bilan de l'utilisation de I'habitat par la truite lors de sa période juvénile en ruisseau. Ce bilan s'applique en premier lieu aux petits cours d'eau peu pentus tels qu'on les trouve dans le Massif Armoricain. Cependant, les éléments bibliographiques cités dans cet article et issus d'autres types de milieux, ainsi que les expérimentations réalisées dans les Pyrénées Atlantiques, apportent de précieuses indications pour élargir la portée des résultats.

Dans ces petits cours d'eau, l'alternance de faciès peu profonds et courants (moins de $20 \mathrm{~cm}$ et plus de $20 \mathrm{~cm} / \mathrm{s}$ ) et de faciès profonds et lents $(40 \mathrm{~cm}$ et plus, moins de $10 \mathrm{~cm} / \mathrm{s}$ ), est une unité d'habitat élémentaire pour le juvénile de truite. Que ce soit à l'échelle nycthémérale ou saisonnière, les changements d'habitat observés au cours du développement ontogénique peuvent y prendre place. Connectées aux migrations à l'échelle de la population, les tendances mises à jour peuvent être résumées ainsi (Figure 6) : 

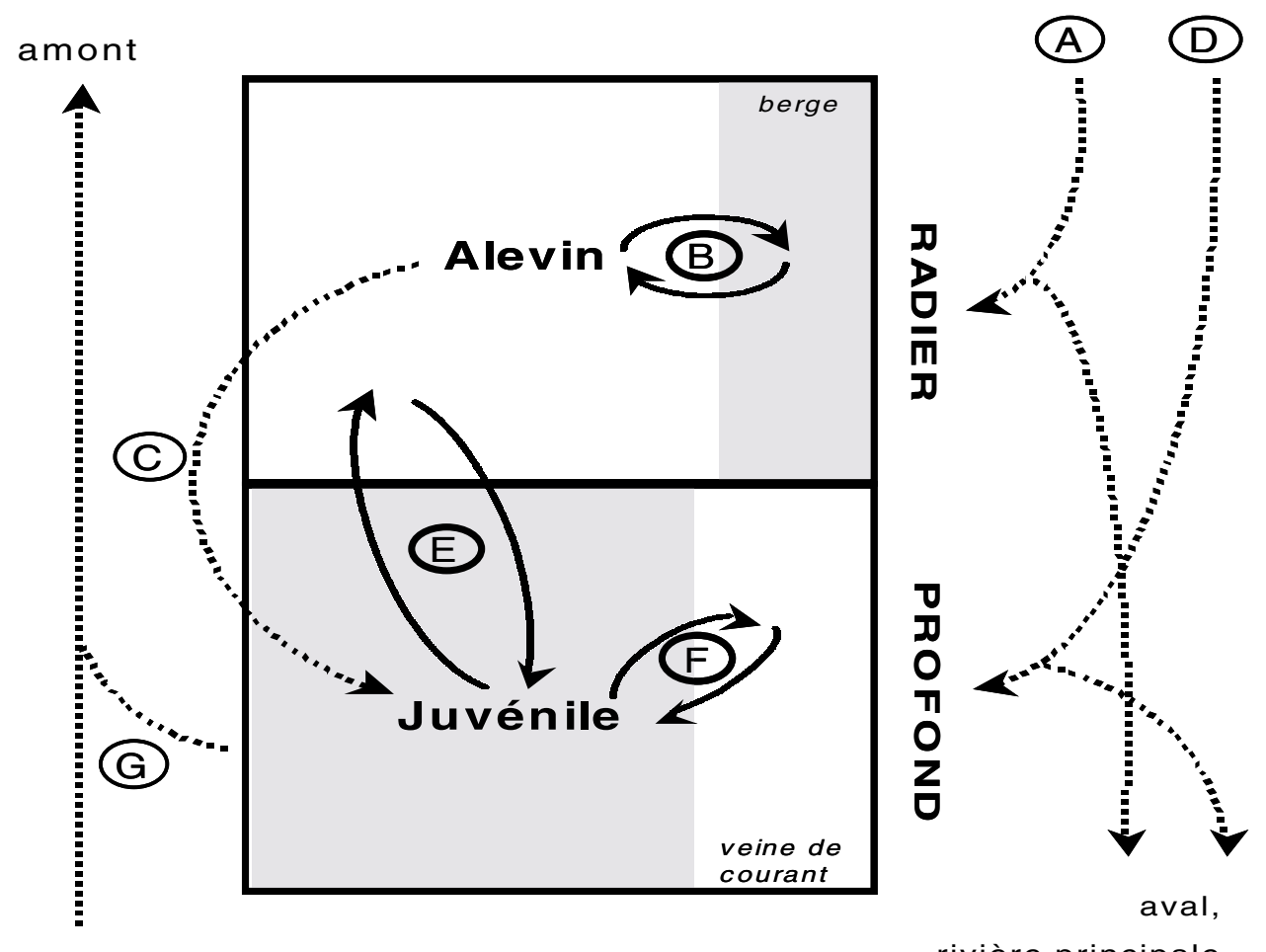

rivière principale

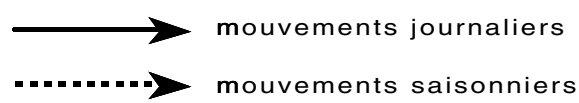

habitat d'alimentation

habitat de repos

Figure 6

Représentation schématique de l'utilisation de l'habitat par la truite durant la période juvénile en ruisseau. On entend par séquence radier/profond l'alternance d'un faciès peu profond $(<20 \mathrm{~cm})$ aux vitesses de courant $>20 \mathrm{~cm} / \mathrm{s}$, et d'un faciès profond $(>40 \mathrm{~cm})$ et lent $(<10 \mathrm{~cm} / \mathrm{s})$. Les lettres désignent les principaux mouvements journaliers (flèches pleines) et saisonniers (flèches pointillées) identifiés chez l'animal : $A=$ dévalaison postémergence en début de printemps ; $B$ = rythme journalier berge/chenal des alevins ; $\mathbf{C}=$ glissement des juvéniles de l'année en direction des profonds ; $\mathbf{D}=$ dispersion des juvéniles d'un été, dès l'hiver et surtout au printemps ; $E$ = rythme journalier radier/profond des juvéniles d'un été et plus ; $F=$ rythme journalier à l'intérieur du profond pour les juvéniles d'un été et plus ; $G=$ migration de reproduction des individus matures (1+ précoces, ou plus âgés), en fin d'automne et début d'hiver. On se référera au texte principal pour plus d'explications (paragraphe "Bilan de l'utilisation de l'habitat par le juvénile de truite en ruisseau $")$.

Figure 6

Schematic diagram that summarizes movements of juvenile brown trout in small tributaries. The riffle/pool unit is characterized by shallow $(<20 \mathrm{~cm})$ and swift water $(>20 \mathrm{~cm} / \mathrm{s}$ ) in riffle (radier), whereas pools (profond) are deeper $(>40 \mathrm{~cm}$ ) and slow flowing $(<10 \mathrm{~cm} / \mathrm{s})$. Grey and white areas correspond to resting and feeding positions, respectively. "Berge " = stream margin habitat; " veine de courant " = highest velocity zone in the pool. From A to G: seasonal (dashed arrows) and diel (solid arrows) movements that are documented. $A=$ post-emergence drift in Spring; $B=$ diel movements of alevins between stream-margin and off-shore habitats; $\mathbf{C}=$ shift from riffle to pool habitats (young-of-the-year); $\mathrm{D}=$ downstream migration of one-summer-old juveniles, mostly in Spring; $\mathrm{E}=$ diel movements between pool and riffle (one-summer-old and older juveniles); $\mathbf{F}=$ diel movements between low and higher velocity microhabitats in the pool (one-summer-old and older juveniles); G = spawning migration upward (precocious oneyear-old fish and older individuals) in late Fall and early Winter. 
Lors de la dévalaison post-émergence décrite par CUINAT et HÉLAND (1979) en début de printemps, les alevins se distribuent près des berges (Figure 6, A), à l'abri du courant et des poissons prédateurs. Le rythme nycthéméral d'utilisation de l'habitat se met rapidement en place, les alevins quittant les berges de jour pour s'alimenter dans des habitats plus courants et profonds en chenal (Figure 6, B). C'est dans les faciès courants de type radier que l'on trouve les plus fortes densités d'alevins, mais les berges des faciès plus profonds peuvent également être utilisées (LAVOIE et HUBERT, 1996).

A mesure qu'ils grandissent, les alevins délaissent les berges et recherchent des habitats plus profonds. Au cours de l'ontogenèse, le passage en faciès profond est progressif (Figure 6, C), les séquences radier/profond offrant souvent un continuum de conditions d'habitat propices (JOWETT, 1993 ; ROUSSEL et BARDONNET, 1996). Une fois dans le profond, il est probable que les juvéniles de l'année $(0+)$ adoptent un comportement proche de celui des juvéniles d'un an et plus, bien que cet aspect reste à approfondir.

Dès l'hiver et surtout au début du printemps, on assiste à la dispersion des juvéniles d'un été (Figure 6, D) depuis les zones d'amont et sous-affluents fortement peuplés vers l'aval du ruisseau ou le cours principal (BAGLINIĖRE et al., 1989). Les juvéniles 1+ entament leur deuxième saison de croissance, et les faciès profonds du ruisseau ont surtout valeur d'habitat de repos et refuge.

A la faveur des rythmes journaliers d'activité, les juvéniles 1+ se distribuent dans les habitats plus rapides en radier (Figure 6, E) ou dans les veines de courant (Figure 6, F) pour s'alimenter sur la dérive. Les mouvements journaliers entre radier et profond sont particulièrement marqués pendant la période estivale, lorsque les débits sont faibles et les postes d'alimentation sur dérive plus rares.

A l'approche du deuxième hiver, les mâles $1+$ précoces vont pouvoir participer, le cas échéant, à la migration des adultes vers l'amont, en direction des zones de reproduction (Figure 6, G) (BAGLINIÈRE et al., 1987). Les autres juvéniles n'atteindront l'âge de reproduction qu'à l'issu d'une voire deux années supplémentaires.

Ainsi, la séquence radier/profond supporte les principales activités et comportements vitaux du juvénile de truite au cours de son développement ontogénique en ruisseau. A ce titre, elle peut être considérée comme une unité fonctionnelle d'habitat pour le juvénile. On notera que BUNNELL et al. (1998) ont également montré l'existence de déplacements nycthéméraux entre radier et profond chez l'adulte, grâce à des techniques de radio-pistage. D'une manière générale, nos résultats soulignent l'importance de l'hétérogénéité de l'habitat dans les petits cours d'eau à truites. Les implications sont importantes en terme de protection des milieux naturels, car les changements d'occupation des sols ou l'intensification des activités humaines sur le bassin versant entraînent souvent la perte de diversité des habitats lotiques (GREGORY et al., 1994 ; WALSER et BART, 1999). Dans ce sens, préserver la diversité fonctionnelle des habitats semble s'imposer comme une règle générale en matière de gestion des milieux trutticoles. L'attention pourra être plus particulièrement portée sur :

les habitats de bordure, peu profonds et abrités le long des berges, nécessaires aux alevins dans les semaines qui suivent l'émergence,

la présence d'abris dans le chenal (blocs, débris ligneux, végétaux aquatiques) favorisant le partage des postes d'alimentation favorables entre les juvéniles,

l'alternance radier-profond en général. On notera que ce niveau d'hétérogénéité est intimement lié à la présence d'obstacles naturels à l'écoulement tels que les bois morts dans le chenal (GREGORY et DAVIS, 1992 ; MONTGOMERY et al., 1995), et aussi à l'altération des berges (MYERS et SWANSON, 1991). 


\section{BIBLIOGRAPHIE}

ALLARDI J., KEITH P., 1991. Atlas préliminaire des poissons d'eau douce de France. Collection patrimoines naturels volume 4, série patrimoine génétique, Muséum National d'Histoire Naturelle, Paris.

BACHMAN R.A., REYNOLDS W.W., CASTERLIN M.E., 1979. Diel locomotor activity patterns of wild brown trout (Salmo trutta L.) in an electronic shuttlebox. Hydrobiologia, 66, 45-47.

BAGLINIËRE J.L., 1979. Les principales populations de poissons sur une rivière à salmonidés de Bretagne-Sud, le Scorff. Cybium, 7, 53-74.

BAGLINIĖRE J.L., CHAMPIGNEULLE A., 1982. Densités des populations de truite commune (Salmo trutta L.) et de juvéniles de saumon atlantique (Salmo salar L.) sur le cours principal du Scorff (Bretagne) : préférendums physiques et variations annuelles (1976-1980). Acta Oecol., 3, 241-256.

BAGLINIĖRE J.L., MAISSE G., LEBAIL P.Y., NIHOUARN A., 1989. Population dynamics of brown trout, Salmo trutta L., in a tributary in Brittany (France): spawning and juveniles. J. Fish Biol., 34, 97-110.

BAGLINIĖRE J.L., MAISSE G., LEBAIL P.Y., PREVOST E., 1987. Dynamique de la population de truite commune (Salmo trutta L.) d'un ruisseau breton (France). II - Les géniteurs migrants. Acta Oecol., 8, 201-215.

BALON E.K., 1975. Terminology of intervals in fish development. J. Fish. Res. Bd Can., 32, 1663-1670.

BARAN P., 1995. Analyse de la variabilité des abondances de truites communes (Salmo trutta L.) dans les Pyrénées centrales françaises. Thèse de troisième cycle, Institut National Polytechnique de Toulouse, $147 \mathrm{p}$.

BARAN P., DELACOSTE M., DAUBA F., LASCAUX J.M., BELAUD A., LEK S., 1995. Effects of reduced flow on brown trout (Salmo trutta L.) populations downstream dams in French Pyrénées. Regul. River., 10, 347-361.

BARAN P., DELACOSTE M., LASCAUX J.M., 1997. Variability of mesohabitat used by brown trout populations in the French central Pyrénées. Trans. Am. Fish. Soc., 126, 747-757.

BARAS E., 1992. Etude des stratégies d'occupation du temps et de l'espace chez le barbeau fluviatile, Barbus barbus (L.). Cahiers d'Ethologie Fondamentale et Appliquée, Animale et Humaine. Collection Enquêtes et Dossiers: 16, vol 12, fascicule 2-3. $442 \mathrm{p}$.

BARAS E., 1997. Environmental determinants of residence area selection by Barbus barbus in the river Ourthe. Aquat. Living Resour., 10, 195-206.

BARDONNET A., GAUDIN P., PERSAT H., 1991. Microhabitats and diel dowstream migration of young grayling (Thymallus thymallus L.). Freshwater Biol., 26, 365-376.

BARDONNET A., GAUDIN P., THORPE J.E., 1993. Diel rhythm of emergence and of first displacement downstream in trout (Salmo trutta), Atlantic salmon (S. salar) and grayling (Thymallus thymallus). J. Fish Biol., 43, 755-762.

BELAUD A., CHAVEROCHE P., LIM P., SABATON C., 1989. Probability-of-use curves applied to brown trout (Salmo trutta fario L.) in rivers of southern France. Regul. River., 3, 321-336.

BISSON P.A., MONTGOMERY D.R., 1996. Valley segments, stream reaches and channel units. In : Methods in Stream Ecology (HAUER F.R., LAMBERTI G.A., Eds.), 23-52. London, Academic Press.

BOHLIN T., 1977. Habitat selection and intercohort competition of juvenile sea-trout Salmo trutta. Oikos, 29, 112-117.

BRIDCUT E.E., GILLER P.S., 1993a. Movement and site fidelity in young brown trout Salmo trutta populations in a southern Irish stream. J. Fish Biol., 43, 889-899.

BRIDCUT E.E., GILLER P.S., 1993b. Diet variability in relation to season and habitat utilisation in brown trout, Salmo trutta L., in a southern Irish stream. Can. Spe. Pub. Fish. Aquat. Sci., 118, 17-24. 
BUNNELL D.B., ISELY J.J., BURRELL K.H., VAN LEAR D.H., 1998. Diel movement of brown trout in a southern Appalachian river. Trans. Am. Fish. Soc., 127, 630-636.

BUTLER R.L., HAWTHORNE V.M., 1968. The reactions of dominant trout to changes in overhead artificial cover. Trans. Am. Fish. Soc., 97, 37-41.

CAPRA H., 1995. Amélioration des modèles prédictifs d'habitat de la truite fario : échelles d'échantillonnage, intégration des chroniques hydrologiques. Thèse de troisième cycle, Université Lyon I, $270 \mathrm{p}$.

CHANGEUX T., 1997. 1955-1997 : 42 ans de subventions du CSP. Eaux libres, 23, 14-15.

CHASTON I., 1968. Influence of light on activity of brown trout (Salmo trutta). J. Fish. Res. Bd Can., 25, 1285-1289.

CHASTON I., 1969. Seasonal activity and feeding pattern of brown trout (Salmo trutta) in a Dartmoor stream in relation to availability of food. J. Fish. Res. Bd Can., 26, 21652171.

CLAPP D.F., CLARK R.D., DIANA J.S., 1990. Range, activity, and habitat of large, freeranging brown trout in a Michigan stream. Trans. Am. Fish. Soc., 119, 1022-1034.

CUINAT R., HÉLAND M., 1979. Observations sur la dévalaison d'alevins de truite commune (Salmo trutta) dans le Lissuraga. Bull. Fr. Pêche Piscic., 274, 2-17.

CUNJAK R.A., POWER G., 1987. Cover use by stream-resident trout in winter: a field experiment. North Am. J. Fish. Manage., 7, 539-544.

DELACOSTE M., 1995. Analyse de la variabilité spatiale de la reproduction de la truite commune (Salmo trutta L.). Etude à l'échelle du micro et du macrohabitat dans six rivières des Pyrénées centrales. Thèse de troisième cycle, Institut National Polytechnique de Toulouse, $132 \mathrm{p}$.

DEVORE P., WHITE R.J., 1978. Daytime responses of brown trout (Salmo trutta) to cover stimuli in stream channels. Trans. Am. Fish. Soc., 107, 763-771.

EGGLISHAW H.J., SHACKLEY P.E., 1982. Influence of water depth on dispersion of juvenile salmonids, Salmo salar L. and Salmo trutta L., in a Scottish stream. J. Fish Biol., 21, 141-155.

ELLIOTT J.M., 1970. Diel changes in invertebrate drift and the food of trout Salmo trutta L. J. Fish Biol., 2, 161-165.

ELLIOTT J.M., 1994. Quantitative ecology and the brown trout (MAY R.M. and HARVEY P.H., Eds.). Oxford University Press, $286 \mathrm{p}$.

ERIKSSON L.O., 1973. Spring inversion of diel rhythm of locomotion activity in young seagoing brown trout, Salmo trutta trutta L., and atlantic salmon, S. Salar L. Aquil. Ser. Zool., 14, 68-79.

ERIKSSON L.O., 1978. Nocturnalism versus diurnalism, dualism within fish individuals. In : Rhythmic activity of fishes (THORPE J.E., ed.). 69-89, Academic Press, London.

EUZENAT G., FOURNEL F., 1976. Recherches sur la truite commune (Salmo trutta L.) dans une rivière de Bretagne : le Scorff. Thèse de troisième cycle, Université de Rennes, $230 \mathrm{p}$.

FACEY D.E., GROSSMAN G.D., 1992. The relationship between water velocity, energetic costs and microhabitat use in four North American stream fishes. Hydrobiologia, 239, 1-6.

FAUSCH K.D., 1984. Profitable stream positions for salmonids : relating specific growth rate to net energy gain. Can. J. Zool., 62, 441-451.

FRAGNOUD E., 1987. Préférences d'habitat de la truite fario (Salmo trutta fario L.) en rivières (quelques cours d'eau du sud de la France). Thèse de troisième cycle, Université de Lyon, 398 p.

GERKING S.D., 1994. Feeding ecology of fish (GERKING S.D., ed.). Academic Press, San Diego.

GINOT V., 1995. EVHA, un logiciel d'évaluation de l'habitat sous Windows. Bull. Fr. Pêche Piscic., 337/338/339, 303-308.

GLOVA G.J., FIELD-DODGSON M.S., 1995. Behavioural interaction between chinook salmon and brown trout juveniles in a simulated stream. Trans. Am. Fish. Soc., 124, 194-206. 
GOSSE J.C., HELM W.T., 1982. A method for measuring microhabitat components for lotic fishes and its application with regard to brown trout. In : Aquisition and Utilization of Aquatic Habitat Inventory Information (ARMANTROUT N.B., ed.), 138-149. Bethesda, American Fisheries Society.

GOURAUD V., 1999. Etude de la dynamique de populations de truite commune (Salmo trutta L.) à l'aide d'un modèle déterministe : application sur un bassin basnormand et sur un bassin pyrénéen. Thèse de troisième cycle, Ecole Nationale du Génie Rural, des Eaux et Forêts, Paris, 254 p.

GRANT J.W.A., KRAMER D.L., 1990. Territory size as a predictor of the upper limit to population density of juvenile salmonids in streams. Can. J. Fish. Aquat. Sci., 47, 1724-1737.

GREGORY K.J., DAVIS R.J., 1992. Coarse woody debris in stream channels in relation to river channel management in woodland areas. Regul. River., 7, 117-136.

GREGORY K.J., GURNELL A.M., HILL C.T., TOOTH S., 1994. Stability of the pool-riffle sequence in changing river channels. Regul. River., 9, 35-43.

HAURY J., BAGLINIĖRE J.L., 1990. Relations entre la population de truites communes (Salmo trutta), les macrophytes et les paramètres du milieu sur un ruisseau. Bull. Fr. Pêche Piscic., 318, 118-131.

HAURY J., BAGLINIĖRE J.L., 1996. Les macrophytes, facteur structurant de l'habitat piscicole en rivière à salmonidés. Etude de microrépartition sur un secteur végétalisé du Scorff (Bretagne-Sud). Cybium, 20 (supplément 3), 111-127.

HAYES J.W., JOWETT I.G., 1994. Microhabitat models of large drift-feeding brown trout in three New Zealand Rivers. North Am. J. Fish. Manage., 14, 710-725.

HEGGENES J., 1988. Physical habitat selection by brown trout (Salmo trutta) in riverine systems. Nord. J. Freshwater Res., 64, 74-90.

HEGGENES J., KROG O.M., LINDAS O.R., DOKK J.G., BREMNES T., 1993. Homeostatic behavioural responses in a changing environment: brown trout (Salmo trutta) become nocturnal during winter. J. Anim. Ecol., 62, 295-308.

HÉLAND M., 1971. Observations sur les premières phases du comportement agonistique et territorial de la truite commune Salmo trutta L. en ruisseau artificiel. Ann/s Hydrobiol., 2, 33-46.

HÉLAND M., 1977. Recherches sur l'ontogenèse du comportement territorial chez l'alevin de truite commune (Salmo trutta L.). Thèse de troisième cycle, Université de Rennes, $239 \mathrm{p}$.

HÉLAND M., GAUDIN P., BARDONNET A., 1995. Mise en place des premiers comportements et utilisation de l'habitat après l'émergence chez les salmonidés d'eau courante. Bull. Fr. Pêche Piscic., 337/338/339, 191-197.

HELFMAN G.S., 1993. Fish behaviour by day, night and twilight. In : Behaviour of teleost fishes (PITCHER T.J., ed.), 479-512. Chapman \& Hall, London.

HILL J., GROSSMAN G.D., 1993. An energetic model of microhabitat use for rainbow trout and rosyside dace. Ecology, 74, 685-698.

HOLL M., 1997. Les raisons d'un virage. Eaux libres, 23, 11-13.

HUBERT W.A., HARRIS D.D., WESCHE T.A., 1994. Diurnal shifts in use of summer habitat by age-0 brown trout in a regulated mountain stream. Hydrobiologia, 284, 147-156.

HUBERT W.A., RHODES H.A., 1992. Sizes of prey consummed by age-0 brown trout in Douglas creek, Wyoming. J. Freshwater Ecol., 7, 277-282.

HUET M., 1954. Biologie, profils en long et en travers des eaux courantes. Revue Suisse d'Hydrobiologie, 11, 332-351.

HUGHES N.F., 1992. Selection of positions by drift-feeding salmonids in dominance hierachies: model and test for Arctic grayling (Thymallus arcticus) in subarctic mountain streams, interior Alaska. Can. J. Fish. Aquat. Sci., 49, 1999-2008.

HUGHES N.F., DILL L.M., 1990. Position choice by drift-feeding salmonids: model and test for arctic grayling (Thymallus arcticus) in subarctic mountain streams, interior Alaska. Can. J. Fish. Aquat. Sci., 47, 2039-2048.

JONES A.N., 1975. A preliminary study of fish segregation in salmon spawning streams. J. Fish Biol., 7, 95-104. 
JOWETT I.G., 1993. A method for objectively identifying pool, run and riffle habitat from physical measurements. New Zeal. J. Mar. Freshwater Res., 27, 241-248.

KARLSTRÖM O., 1977. Habitat selection and population densities of salmon (Salmo salar L.) and trout (Salmo trutta L.) parr in Swedish rivers with some reference to human activities. Acta Universitatis Upsaliensis, 404, $12 \mathrm{p}$.

KEELEY E.R., GRANT J.W.A., 1995. Allometric and environmental correlates of territory size in juvenile Atlantic salmon (Samo salar). Can. J. Fish. Aquat. Sci., 52, 186-196.

KENNEDY G.J., STRANGE C.D., 1986. The effects of intra- and inter-specific competition on the distribution of stocked juvenile Atlantic salmon, Salmo salar L., in relation to depth and gradient in an upland trout, Salmo trutta, stream. J. Fish Biol., 29, 199-214.

KOCIK J.F., TAYLOR W.W., 1996. Effect of juvenile Steelhead on juvenile brown trout habitat use in a low-gradient Great Lakes tributary. Trans. Am. Fish. Soc., 125, 244252.

LAMOUROUX N., 1997. Hydraulique statistique et prédiction des caractéristiques du peuplement piscicole : modèles pour l'écosystème fluvial. Thèse de troisième cycle, Université Lyon I, 38 p. + annexes.

LAVOIE W.J., HUBERT W.A., 1996. Use of three types of stream-margin habitat by age-0 brown trout late in the growing season. Hydrobiologia, 317, 89-95.

LÉGER L., 1910. Principes de la méthode rationnelle du peuplement des cours d'eau à salmonidés. Travaux du Laboratoire de Pisciculture de I'Université de Grenoble, 1910, 531-568.

LEWIS S., 1969. Physical factors influencing fish populations in pools of a trout stream. Trans. Am. Fish. Soc., 98, 14-19.

LIM P., SEGURA G, BELAUD A., SABATON C., 1993. Etude de l'habitat de la truite fario (Salmo trutta fario). Rôle des caches artificielles et naturelles dans les rivières aménagées. Bull. Fr. Pêche Piscic., 331, 373-396.

MacARTHUR R.H., PIANKA E.R., 1966. On optimal use of a patchy environment. Am. Nat., 100, 603-609.

MÄKI-PETÄYS A., MUOTKA T., HUUSKO A., TIKKANEN P., KREIVI P., 1997. Seasonal changes in habitat use and preference by juvenile brown trout, Salmo trutta, in a northern boreal river. Can. J. Fish. Aquat. Sci., 54, 520-530.

MARIDET L., SOUCHON Y., 1995. Habitat de la truite fario (Salmo trutta fario L.) dans trois cours d'eau du Massif Central ; approche méthodologique et premiers résultats sur le rôle de la végétation rivulaire arborée. Bull. Fr. Pêche Piscic., 336, 1-18.

MESICK C., 1988. Effects of food and cover on numbers of apache and brown trout establishing residency in artificial sream channels. Trans. Am. Fish. Soc., 117, 421-431.

MONTGOMERY D.R., BUFFINGTON J.M., SMITH R.D., SCHMIDT K.M., PESS G., 1995. Pool spacing in forest channels. Water Resour. Res., 31, 1097-1105.

MOORE K.M., GREGORY S.V., 1988. Summer habitat utilization and ecology of cutthroat trout fry (Salmo clarki) in cascade mountain streams. Can. J. Fish. Aquat. Sci., 45, 1921-1930.

MORTENSEN E., 1977. Density-dependent mortality of trout fry (Salmo trutta L.) and its relationship to the management of small streams. J. Fish Biol., 11, 613-617.

MÜLLER K., 1978. Locomotor activity of fish and environmental oscillations. In : Rhythmic activity of fishes (THORPE J.E., ed.). 1-19, Academic Press, London.

MYERS T.J., SWANSON S., 1991. Aquatic habitat condition index, stream type, and livestock bank damage in northern Nevada. Water Resour. Bull., 27, 667-677.

NEVEU A., 1979. Les rythmes alimentaires en milieu naturel. In : Nutrition des poissons (FONTAINE M., ed.). Actes du colloque CNERMA, 339-353, Paris.

NIELSEN G., 1986. Dispersion of brown trout (Salmo trutta L.) in relation to stream cover and water depth. Pol. Arch. Hydrobiol., 33, 475-488.

NIHOUARN A., 1983. Etude de la truite commune (Salmo trutta L.) dans le bassin du Scorff (Morbihan) : démographie, reproduction, migrations. Thèse de troisième cycle, Université de Rennes, 64 p. 
OVIDIO M., 1999. Tactiques et stratégies individuelles d'utilisation spatio-temporelle de l'habitat et des ressources alimentaires chez la truite commune (Salmo trutta L.) : étude par radio-pistage dans l'Aisne et l'Ourthe. Thèse de troisième cycle, Université de Liège, $196 \mathrm{p}$.

PEREZ E., 1986. Rôle des facteurs externes et internes dans la mise en place du rythme circadien d'activité au cours de l'ontogenèse de la truite (Salmo trutta L.). Thèse de troisième cycle, Université de Saint-Etienne, $283 \mathrm{p}$.

POUILLY M., VALENTIN S., CAPRA H., GINOT V., SOUCHON Y., 1995. Méthode des microhabitats : principes et protocoles d'application. Bull. Fr. Pêche Piscic., 336, 41-54.

ROBINSON F.W., TASH J.C., 1979. Feeding by Arizona trout (Salmo apache) and brown trout (Salmo trutta) at different light intensities. Environ. Biol. Fish, 4, 363-368.

ROUSSEL J.M., BARDONNET A., 1995. Activité nycthémérale et utilisation de la séquence radier/profond par les truitelles d'un an (Salmo trutta L.). Bull. Fr. Pêche Piscic., 337/338/339, 221-230.

ROUSSEL J.M., BARDONNET A., 1996. Changements d'habitat de la truite (Salmo trutta) et du chabot (Cottus gobio) au cours du nycthémère : approches multivariées à différentes échelles spatiales. Cybium, 20 (supplément 3), 43-53.

ROUSSEL J.M., BARDONNET A., 1997. Diel and seasonal patterns of habitat use by fish in a natural salmonid brook : an approach to the functional role of the riffle-pool sequence. Bull. Fr. Pêche Piscic., 346, 573-588.

ROUSSEL J.M., 1998. Utilisation de l'espace par la truite commune (Salmo trutta L.) au cours du nycthémère. Thèse de troisième cycle, Ecole Nationale Supérieure Agronomique de Rennes, $186 \mathrm{p}$.

ROUSSEL J.M., BARDONNET A., 1999. Ontogeny of diel pattern of stream-margin habitat use by emerging brown trout (Salmo trutta L.) in experimental channels: influence of food and predator presence. Environ. Biol. Fish, 56, 253-262.

ROUSSEL J.M., BARDONNET A., CLAUDE A., 1999. Microhabitats of brown trout when feeding on drift and when resting in a lowland salmonid brook: effects on Weighted Usable Area. Arch. Hydrobiol., 146, 413-429.

SHIRVELL C.S., DUNGEY R.G., 1983. Microhabitat chosen by brown trout for feeding and spawning in rivers. Trans. Am. Fish. Soc., 112, 355-367.

SHULER S.W., NEHRING R.B., 1994. Using the physical habitat simulation model to evaluate a stream habitat enhancement project. Rivers, 4, 175-193.

SIEGEL S., CASTELLAN N.J., 1988. Nonparametric statistics for the behavioural sciences (SIEGEL S. and CASTELLAN N.J., Eds.). Second edition, McGraw-Hill Inc., 399 p.

SOLOMON D.J., TEMPLETON R.G., 1976. Movements of brown trout Salmo trutta L. in a chalk stream. J. Fish Biol., 9, 411-423.

SOUCHON Y., TROCHERIE F., FRAGNOUD E., LACOMBE C., 1989. Les modèles numériques des microhabitats des poissons : application et nouveaux développements. Revue des Sciences de l'Eau, 2, 807-830.

STEINGRIMSSON S.O., GRANT J.W.A., 1999. Allometry of territory size and metabolic rate as predictors of self-thinning in young-of-the-year atlantic salmon. J. Anim. Ecol., 68, 17-26.

SWIFT D.R., 1964. Activity cycles in the brown trout (Salmo trutta L.). 2- Fish artificially fed. J. Fish. Res. Bd Can., 21, 133-138.

VALENTIN S., LAUTERS F., SABATON C., BREIL P., SOUCHON Y., 1996. Modelling temporal variations of physical habitat for brown trout (Salmo trutta) in hydropeaking conditions. Regul. River., 12, 317-330.

WALSER C.A., BART H.L., 1999. Influence of agriculture on in-stream habitat and fish community structure in piedmont watersheds of the Chattahoochee river system. Ecol. Freshwater Fish, 8, 237-246.

WALSH G., MORIN R., NAIMAN R.J., 1988. Daily rations, feeding activity and distribution of age-0 brook charr, Salvelinus fontinalis, in two subarctic streams. Environ. Biol. Fish, 21, 195-205. 
WESCHE T.A., GOERTLER C.M., FRYE C.B., 1987. Contribution of riparian vegetation to trout cover in small streams. North Am. J. Fish. Manage., 7, 151-153.

YOUNG M.K., 1999. Summer diel activity and movement of adult brown trout in highelevation streams in Wyoming, USA. J. Fish Biol., 54, 181-189.

YOUNG M.K., WILKISON R.A., PHELPS J.M., GRIFFITH J.S., 1997. Contrasting movement and activity of large brown trout and rainbow trout in Silver Creek, Idaho. Great Basin Nat., 57, 238-244.

ZIPPIN C., 1956. An evaluation of the removal method of estimating animal populations. Biometrics, 12, 163-189. 